Orbett T. Alexander* and Johan Venter

\title{
Crystal structure of monocarbonyl( $\mathrm{N}$-nitroso- $\mathrm{N}$ - oxido-phenylamine- $\kappa^{2} O, O^{\prime}$ ) (tricyclohexylphosphine-kP)rhodium(I), $\mathrm{C}_{25} \mathrm{H}_{39} \mathrm{~N}_{2} \mathrm{O}_{3} \mathrm{PRh}$
}

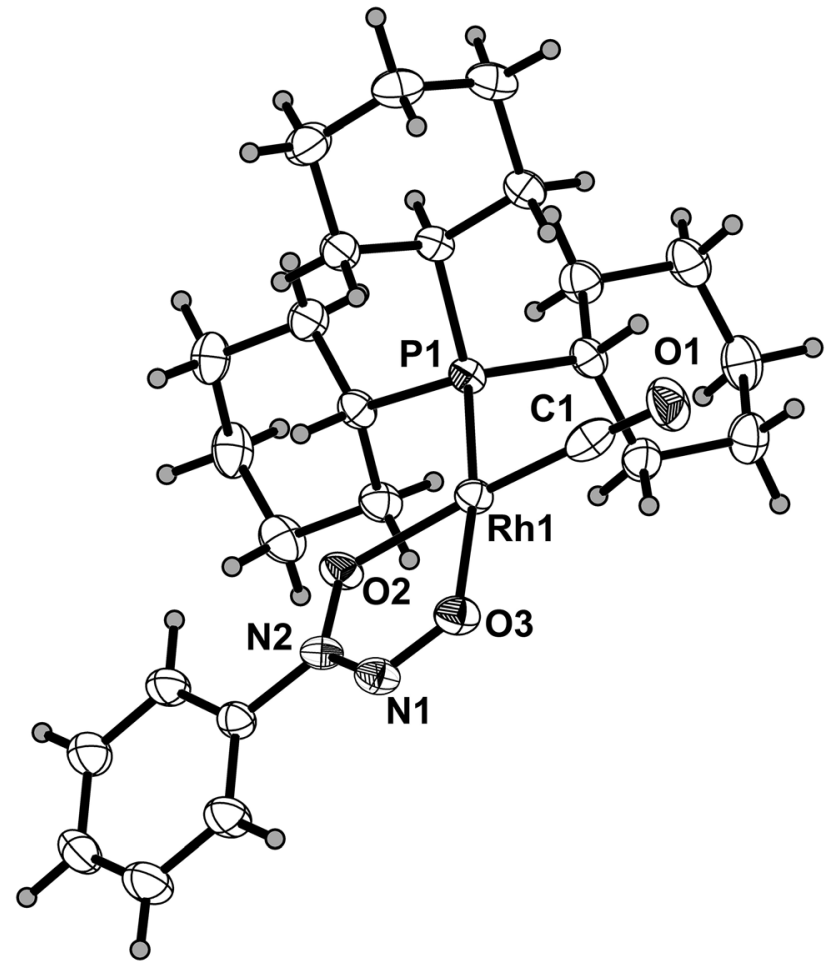

https://doi.org/10.1515/ncrs-2021-0218

Received May 31, 2021; accepted June 17, 2021; published online July 19, 2021

\section{Abstract \\ $\mathrm{C}_{25} \mathrm{H}_{39} \mathrm{~N}_{2} \mathrm{O}_{3} \mathrm{PRh}$, triclinic, $P 2_{1} / n$ (no. 14), $a=13.505$ (5) $\AA$, $b=14.266(5) \AA, \quad c=13.405(4) \AA, \quad \beta=91.280(3)^{\circ}$, $V=2574.8(15) \AA^{3}, Z=4, R_{g t}(F)=0.0399, w R_{\text {ref }}\left(F^{2}\right)=0.1026$, $T=100 \mathrm{~K}$.}

CCDC no.: 2086956
Table 1: Data collection and handling.

\begin{tabular}{|c|c|}
\hline Crystal: & Orange plate \\
\hline Size: & $0.24 \times 0.19 \times 0.10 \mathrm{~mm}$ \\
\hline Wavelength: & Mo $K \alpha$ radiation $(0.71073 \AA$ ) \\
\hline$\mu$ : & $0.75 \mathrm{~mm}^{-1}$ \\
\hline Diffractometer, scan mode: & Bruker APEX-II, $\varphi$ and $\omega$ \\
\hline$\theta_{\max }$, completeness: & $28.0^{\circ}, 99 \%$ \\
\hline$N(h k l)_{\text {measured }}, N(h k l)_{\text {unique }}, R_{\text {int }}:$ & $31,008,6189,0.070$ \\
\hline Criterion for $I_{\mathrm{obs}}, N\left(h k l_{\mathrm{gt}}\right.$ : & $I_{\text {obs }}>2 \sigma\left(I_{\text {obs }}\right), 5256$ \\
\hline$N(\text { param })_{\text {refined: }}$ & 289 \\
\hline Programs: & $\begin{array}{l}\text { Bruker [1], SIR97 [2], WinGX [3], } \\
\text { SHELX [4], Diamond [5] }\end{array}$ \\
\hline
\end{tabular}

The molecular structure is shown in the figure. Table 1 contains crystallographic data and Table 2 contains the list of the atoms including atomic coordinates and displacement parameters.

\section{Source of material}

The synthesis of the title complex started with refluxing of hydrated $\mathrm{RhCl}_{3}$ in DMF for 30 min [6]. After cooling, an equivalent amount of the ammonium salt of $\mathrm{N}$-nitrosophenylhydroxylamine (cupferron) was added to the DMF solution [7], whereupon [ $\mathrm{Rh}(\mathrm{cupf})(\mathrm{CO})_{2}$ ] was precipitated by the addition of ice-water. $\left[\mathrm{Rh}(\mathrm{cupf})(\mathrm{CO})\left(\mathrm{PCy}_{3}\right)\right]$ was prepared by adding an equivalent amount of tricyclohexylphosphine to an acetone solution of $\left[\mathrm{Rh}(\mathrm{cupf})(\mathrm{CO})_{2}\right]$ and precipitated with the addition of ice-water. Orange single crystals of $\left[\mathrm{Rh}\right.$ (cupf)(CO)( $\left.\left.\mathrm{PCy}_{3}\right)\right]$ suitable for data collection were obtained after recrystallization from a 1:1 dichloromethane acetonitrile mixture.

\section{Experimental details}

The $\mathrm{H}$ atoms were placed in geometrically idealized positions and constrained to ride on their parent atoms, with C$\mathrm{H}=0.95$ and $0.98 \AA$ and $U_{\text {iso }}(\mathrm{H})=1.5$ and $1.2 U_{\text {eq }}(\mathrm{C})$, Chemistry, University of the Free State, Bloemfontein, 9301, South Africa, E-mail: alexanderorbett@gmail.com. https://orcid.org/00000003-4926-8342

Johan Venter, Department of Chemistry, University of the Free State, Bloemfontein, 9301, South Africa 
Table 2: Fractional atomic coordinates and isotropic or equivalent isotropic displacement parameters $\left(\AA^{2}\right)$.

\begin{tabular}{|c|c|c|c|c|}
\hline Atom & $x$ & $y$ & $z$ & $U_{\text {iso }}{ }^{*} / U_{\text {eq }}$ \\
\hline Rh1 & $0.27045(2)$ & $0.23037(2)$ & $0.40595(2)$ & $0.02524(8)$ \\
\hline $\mathrm{P} 1$ & $0.22497(5)$ & $0.36301(5)$ & $0.32757(5)$ & 0.02454 (15) \\
\hline 01 & $0.08193(18)$ & 0.22858 (19) & $0.5150(2)$ & $0.0480(6)$ \\
\hline 02 & 0.40484 (15) & $0.22150(14)$ & $0.33833(16)$ & $0.0312(4)$ \\
\hline 03 & $0.33146(15)$ & $0.10746(15)$ & $0.46387(16)$ & $0.0328(5)$ \\
\hline N1 & $0.41959(18)$ & 0.08689 (18) & $0.4324(2)$ & $0.0315(5)$ \\
\hline $\mathrm{N} 2$ & $0.45498(17)$ & $0.14612(16)$ & $0.37090(18)$ & $0.0279(5)$ \\
\hline $\mathrm{C} 1$ & $0.1568(3)$ & $0.2303(2)$ & $0.4703(2)$ & $0.0352(7)$ \\
\hline C11 & $0.3319(2)$ & $0.4031(2)$ & $0.2527(2)$ & $0.0284(6)$ \\
\hline $\mathrm{H} 11$ & 0.360617 & 0.344279 & 0.224364 & $0.034^{*}$ \\
\hline $\mathrm{C} 12$ & $0.4162(2)$ & $0.4465(2)$ & $0.3171(2)$ & $0.0323(6)$ \\
\hline $\mathrm{H} 12 \mathrm{~A}$ & 0.393850 & 0.507222 & 0.344712 & $0.039^{\star}$ \\
\hline $\mathrm{H} 12 \mathrm{~B}$ & 0.432213 & 0.404030 & 0.373754 & $0.039^{*}$ \\
\hline C13 & $0.5095(2)$ & $0.4623(3)$ & $0.2558(3)$ & $0.0406(7)$ \\
\hline $\mathrm{H} 13 \mathrm{~A}$ & 0.560806 & 0.493642 & 0.297887 & 0.049 * \\
\hline H13B & 0.536006 & 0.400923 & 0.234188 & $0.049^{*}$ \\
\hline C14 & $0.4859(3)$ & $0.5229(3)$ & $0.1645(3)$ & $0.0420(8)$ \\
\hline $\mathrm{H} 14 \mathrm{~A}$ & 0.467057 & 0.586771 & 0.186359 & $0.050^{*}$ \\
\hline H14B & 0.545735 & 0.528427 & 0.123590 & $0.050^{*}$ \\
\hline C15 & $0.4019(2)$ & $0.4808(2)$ & $0.1016(2)$ & $0.0387(7)$ \\
\hline H15A & 0.423637 & 0.419790 & 0.074103 & $0.046^{*}$ \\
\hline H15B & 0.386418 & 0.523222 & 0.044876 & $0.046^{*}$ \\
\hline C16 & $0.3085(2)$ & $0.4657(2)$ & $0.1619(2)$ & $0.0324(6)$ \\
\hline $\mathrm{H} 16 \mathrm{~A}$ & 0.256715 & 0.435616 & 0.119336 & $0.039^{*}$ \\
\hline H16B & 0.282879 & 0.527128 & 0.184603 & $0.039^{\star}$ \\
\hline $\mathrm{C} 21$ & $0.1807(2)$ & 0.45819 (19) & $0.4090(2)$ & $0.0279(6)$ \\
\hline $\mathrm{H} 21$ & 0.115301 & 0.435915 & 0.433321 & $0.034^{\star}$ \\
\hline $\mathrm{C} 22$ & $0.2449(2)$ & $0.4734(2)$ & $0.5041(2)$ & $0.0348(6)$ \\
\hline $\mathrm{H} 22 \mathrm{~A}$ & 0.255241 & 0.412602 & 0.538606 & $0.042^{*}$ \\
\hline $\mathrm{H} 22 \mathrm{~B}$ & 0.310472 & 0.498307 & 0.485712 & $0.042^{\star}$ \\
\hline $\mathrm{C} 23$ & $0.1948(3)$ & $0.5423(2)$ & $0.5738(3)$ & $0.0412(7)$ \\
\hline $\mathrm{H} 23 \mathrm{~A}$ & 0.132411 & 0.514342 & 0.597393 & $0.049^{*}$ \\
\hline $\mathrm{H} 23 \mathrm{~B}$ & 0.238596 & 0.553662 & 0.632839 & $0.049^{*}$ \\
\hline $\mathrm{C} 24$ & $0.1723(3)$ & $0.6350(2)$ & $0.5224(3)$ & $0.0422(8)$ \\
\hline $\mathrm{H} 24 \mathrm{~A}$ & 0.235272 & 0.666357 & 0.505729 & $0.051^{*}$ \\
\hline $\mathrm{H} 24 \mathrm{~B}$ & 0.136455 & 0.676504 & 0.568655 & $0.051^{*}$ \\
\hline $\mathrm{C} 25$ & $0.1100(3)$ & $0.6214(2)$ & $0.4276(3)$ & $0.0406(7)$ \\
\hline $\mathrm{H} 25 \mathrm{~A}$ & 0.101242 & 0.682708 & 0.393582 & $0.049^{\star}$ \\
\hline $\mathrm{H} 25 \mathrm{~B}$ & 0.043711 & 0.597780 & 0.445138 & $0.049^{*}$ \\
\hline $\mathrm{C} 26$ & $0.1586(2)$ & $0.5519(2)$ & $0.3565(2)$ & $0.0349(7)$ \\
\hline $\mathrm{H} 26 \mathrm{~A}$ & 0.220998 & 0.579132 & 0.332124 & $0.042^{*}$ \\
\hline $\mathrm{H} 26 \mathrm{~B}$ & 0.113892 & 0.540805 & 0.298178 & $0.042^{\star}$ \\
\hline C31 & $0.1233(2)$ & $0.3461(2)$ & $0.2333(2)$ & $0.0269(6)$ \\
\hline H31 & 0.120302 & 0.403498 & 0.190231 & $0.032^{\star}$ \\
\hline $\mathrm{C} 32$ & $0.0204(2)$ & $0.3325(2)$ & $0.2789(2)$ & $0.0345(7)$ \\
\hline $\mathrm{H} 32 \mathrm{~A}$ & 0.022178 & 0.277505 & 0.324251 & $0.041^{*}$ \\
\hline H32B & 0.003460 & 0.388608 & 0.318756 & $0.041^{*}$ \\
\hline $\mathrm{C} 33$ & $-0.0597(2)$ & $0.3171(3)$ & $0.1971(3)$ & $0.0416(8)$ \\
\hline H33A & -0.124296 & 0.305873 & 0.228553 & $0.050^{*}$ \\
\hline H33B & -0.065864 & 0.374514 & 0.155708 & $0.050^{*}$ \\
\hline C34 & $-0.0352(2)$ & $0.2342(2)$ & 0.1309 (3) & $0.0403(8)$ \\
\hline H34A & -0.085601 & 0.229181 & 0.076344 & $0.048^{\star}$ \\
\hline H34B & -0.037070 & 0.175544 & 0.170485 & $0.048^{*}$ \\
\hline C35 & $0.0674(3)$ & $0.2457(3)$ & $0.0863(3)$ & $0.0413(8)$ \\
\hline H35A & 0.066306 & 0.299467 & 0.039428 & $0.050^{*}$ \\
\hline
\end{tabular}

Table 2: (continued)

\begin{tabular}{lrrrr}
\hline Atom & $\boldsymbol{x}$ & $\boldsymbol{y}$ & $\boldsymbol{z}$ & $\boldsymbol{U}_{\text {iso }} \boldsymbol{U}_{\text {eq }}$ \\
\hline H35B & 0.083766 & 0.188436 & 0.048047 & $0.050^{*}$ \\
C36 & $0.1480(2)$ & $0.2623(2)$ & $0.1669(2)$ & $0.0319(6)$ \\
H36A & 0.155116 & 0.205194 & 0.208727 & $0.038^{*}$ \\
H36B & 0.212108 & 0.273756 & 0.134643 & $0.038^{\star}$ \\
C41 & $0.5559(2)$ & $0.1349(2)$ & $0.3381(2)$ & $0.0279(6)$ \\
C42 & $0.5885(2)$ & $0.1907(2)$ & $0.2613(2)$ & $0.0355(7)$ \\
H42 & 0.544764 & 0.233238 & 0.228065 & $0.043^{*}$ \\
C43 & $0.6869(2)$ & $0.1833(3)$ & $0.2334(3)$ & $0.0428(8)$ \\
H43 & 0.710497 & 0.221407 & 0.180847 & $0.051^{*}$ \\
C44 & $0.7504(2)$ & $0.1214(3)$ & $0.2813(3)$ & $0.0438(8)$ \\
H44 & 0.817658 & 0.117649 & 0.262491 & $0.053^{*}$ \\
C45 & $0.7161(3)$ & $0.0647(3)$ & $0.3567(3)$ & $0.0458(8)$ \\
H45 & 0.759559 & 0.020912 & 0.388487 & $0.055^{*}$ \\
C46 & $0.6180(2)$ & $0.0714(2)$ & $0.3865(3)$ & $0.0381(7)$ \\
H46 & 0.594376 & 0.033162 & 0.438986 & $0.046^{*}$ \\
\hline
\end{tabular}

respectively. The highest peak is 2.06 e $\AA^{-3}$ and deepest hole is -1.05 e $\AA^{-3}$.

\section{Comment}

The use of tertiary phosphine mono-dentate ligands in the chemistry of $[\mathrm{Rh}(\mathrm{L}, \mathrm{L}-\mathrm{Bid})(\mathrm{CO})]_{2}$ (where $L, L-\mathrm{Bid}=$ monoanionic bidentate ligands) to yield $\left[\mathrm{Rh}(\mathrm{L}, \mathrm{L}-\mathrm{Bid})(\mathrm{CO})\left(\mathrm{PR}_{3}\right)\right]$ has been extensively studied as potential catalysts precursors [8-11]. Tertiary phosphines are known to increase electron density on the metal centre. As a result, it has the potential to alter the chemistry thereof, depending on the $\mathrm{PR}_{3}[10,12,13]$. Moreover, the introduction of tertiary phosphines not only effect the electronic properties of these systems, but it also brings additional stability to these complexes [10].

More often than not, in square planar complexes of $[\mathrm{Rh}(\mathrm{L}, \mathrm{L}-\mathrm{Bid})(\mathrm{CO})]_{2}$, substitution of tertiary phosphines tends to form two isomers as only one phosphine substitutes a carbonyl. Carbonyls extend the possibilities during the oxidative addition of alkyl iodides since they allow the formation of acyl adducts via migratory insertion into the metal alkyl bonds formed. The major isomer of the substitution product becomes quite predominant when the incoming $\mathrm{PR}_{3}$ ligand is positioned trans to the stronger donor atom of the bidentate ligand. However, in cases where both donors have comparable donor capabilities the isomers will therefore form in comparable ratios [14]. The title structure consists of an $O, O$-donor bidentate ligand (cupferrate), a carbonyl and $\mathrm{PCy}_{3}$ tertiary phosphine trans to the nitroso group. This substitution pattern in $\left[\mathrm{Rh}(\mathrm{cupf})(\mathrm{CO})\left(\mathrm{PCy}_{3}\right)\right]$ is identical to what was 
found in $\left[\mathrm{Rh}\right.$ (cupf)(CO) $\left.\left(\mathrm{PPh}_{3}\right)\right]$ [11] and the related [Rh(neocupf)(CO) $\left.\left(\mathrm{PPh}_{3}\right)\right]$ [15]. Substitution of the carbonyl trans to the nitroso group shows that the nitroso oxygen (O3) has a greater trans influence than $\mathrm{O} 2$.

The geometric coordination is that of a square planar one. However, the square planar geometric orientation around the rhodium atom is significantly distorted from the ideal geometry, mainly caused by the small $\mathrm{O} 2-\mathrm{Rh}-03$ bite angle $\left(76.45(8)^{\circ}\right)$ of the cupferrate ligand. This angular distortion is also indicated by the $\mathrm{C} 1-\mathrm{Rh}-\mathrm{O} 2$ angle of $175.52(11)^{\circ}$. The respective $\mathrm{Rh}-\mathrm{O}$ bond lengths are 2.075(2) and 2.051(6) Å which is in agreement with other rhodium(I) complexes with 0,0-donor atoms [11, 14-16].

Author contributions: All the authors have accepted responsibility for the entire content of this submitted manuscript and approved submission.

Research funding: National Research Foundation of South Africa (Grant UID: 116302) and University of the Free State.

Conflict of interest statement: The authors declare no conflicts of interest regarding this article.

\section{References}

1. Bruker. SAINT-Plus (version 7.12); Bruker AXS Inc.: Madison, Wisconsin, USA, 2004.

2. Altomare A., Burla M. C., Camalli M., Cascarano G. L., Giacovazzo C., Guagliardi A., Moliterni A. G. G., Polidori G., Spagna R. SIR97: a new tool for crystal structure determination and refinement. J. Appl. Crystallogr. 1999, 32, 115-119.

3. Farrugia L. J. WinGX suite for small-molecule single-crystal crystallography. J. Appl. Crystallogr. 1999, 32, 837-838.

4. Sheldrick G. M. A short history of SHELX. Acta Crystallogr. 2008, A64, 112-122.

5. Brandenbug K., Putz H. DIAMOND. Visual Crystal Structure Information System, (version 3.0c); Crystal Impact GbR: Bonn, Germany, 2005.

6. Varshavskii V. S., Cherkasova T. G. A simple method for preparing acetylacetonatodicarbonylrhodium(I). Russ. J. Inorg. Chem. 1967, 12, 899.
7. Goswami K., Singh M. M. Di and monocarbonyl complexes of rhodium(I) containing singly charged bidentate ligands. Transition Met. Chem. 1980, 5, 83-85.

8. Warsink S., Fessha F. G., Purcell W., Venter J. A. Synthesis and characterization of rhodium(I) 2-methylcupferrate complexes and their kinetic behaviour in iodomethane oxidative addition. J. Organomet. Chem. 2013, 726, 14-20.

9. Roodt A., Visser H. G., Brink A. Structure/reactivity relationships and mechanism from X-ray data and spectroscopic kinetic analysis. Chem. Rev. 2011, 17, 241-280.

10. Warsink S., Riekert Kotze P. D., Janse van Rensburg J. M., Venter J. A., Otto S., Botha E., Roodt A. Kinetic-mechanistic and solid-state study of the oxidative addition and migratory insertion of iodomethane to [rhodium( $\mathrm{S}, \mathrm{O}-\mathrm{BdiPT}$ or $\mathrm{N}, \mathrm{O}-$ ox)(CO) $\left.\left(P R^{1} R^{2} R^{3}\right)\right]$ complexes. Eur. J. Inorg. Chem. 2018, 32, 3615-3625.

11. Basson S. S., Leipoldt J. G., Roodt A., Venter J. A. Crystal structure of carbonyl( $N$-hydroxy- $N$-nitroso-benzenaminato- $\left.0,0^{\prime}\right)$ triphenylphosphinerhodium(I). Inorg. Chim. Acta 1986, 118, L45-L47.

12. Otto S., Alexander O. T., Roodt A. Structure and reactivity relationships in trans- $[\mathrm{PtPh}(\mathrm{L}) 2 \mathrm{Cl}]$ as observed from $\mathrm{Cl}$ - anation by $\mathrm{I}-$ upon interchanging phosphine, arsine and stibine (L) ligands. S. Afr. J. Sci. Tech. 2019, 38, 1-15.

13. Manicum A. L. E., Schutte-Smith M., Alexander O. T., Twigge L., Roodt A., Visser H. G. First kinetic data of the $\mathrm{CO}$ substitution in fac- $\left[\operatorname{Re}\left(L, L^{\prime}-\mathrm{Bid}\right)(\mathrm{CO}) 3(\mathrm{X})\right]$ complexes $\left(L, L^{\prime}-\mathrm{Bid}=\right.$ acacetyl acetonate or tropolonate) by tertiary phosphines PTA and PPh3: synthesis and crystal structures of water-soluble rhenium(I) triand dicarbonyl complexes with 1,3,5-triaza7-phosphaadamantane (PTA). Inorg. Chem. Commun. 2019, 101, 93-98.

14. Elmakki M. A. E., Alexander O. T., Venter G. J. S., Venter J. A., Roodt A. Synthesis and structural determination of $[\mathrm{Rh}$ (opo)(CO)(PR $)$ ] complexes (opo- = 2-oxopyridin-1-olate) and in situ isomeric behavior from preliminary kinetic study of iodomethane oxidative addition. J. Coord. Chem. 2021, 74, 444-466.

15. Venter J. A., Purcell W., Visser H. G., Muller T. J. Carbonyl( $N$ nitroso- $\mathrm{N}$-oxido-1-naphtylamine- $\mathrm{K}^{2} \mathrm{O}, \mathrm{O}^{\prime}$ )(triphenylphosphine-kP) rhodium(I) acetone solvate. Acta Crystallogr. 2009, E65, m1578-m1579.

16. Elmakki M. A. E., Alexander O. T., Venter J. A., Roodt A. Crystal structure of carbonyl(2-oxopyridin-1(2H)-olato-k20, $0^{\prime}$ ) (triphenylarsine-KAs)rhodium(I). Z. Kristallogr. N. Cryst. Struct. 2021, 236, 223-225. 